\title{
Runge-Kutta Theory for Volterra Integral Equations of the Second Kind
}

\author{
By H. Brunner, E. Hairer and S. P. Norsett
}

\begin{abstract}
The present paper develops the theory of general Runge-Kutta methods for Volterra integral equations of the second kind. The order conditions are derived by using the theory of $P$-series, which for our problem reduces to the theory of $V$-series. These results are then applied to two special classes of Runge-Kutta methods introduced by Pouzet and by Bel'tyukov.
\end{abstract}

1. Introduction. Consider the (nonlinear) Volterra integral equation of the second kind,

$$
y(x)=f(x)+\int_{a}^{x} K(x, s, y(s)) d s, \quad x \in I:=[a, b] .
$$

We assume that the kernel $K$ is (at least) continuous on $S \times R^{n}, S:=\{(x, s)$ : $a \leqslant s \leqslant x \leqslant b\}$, and that the solution $y$ exist uniquely and is continuous on $I$.

In order to introduce the discretization of (1.1) by (implicit or explicit) RungeKutta methods, let $x_{n}=a+n h, n=0,1, \ldots, N$, with $h=(b-a) / N(N \geqslant 1)$, and denote by $y_{n}$ any approximation to $y\left(x_{n}\right)$. Furthermore, define

$$
F_{n}(x):=f(x)+\int_{a}^{x_{n}} K(x, s, y(s)) d s, \quad x \geqslant x_{n}(n=0,1, \ldots, N-1),
$$

and let $\tilde{F}_{n}(x)$ be an approximation to $F_{n}(x)$. An $m$-stage (implicit) Runge-Kutta method for (1.1) is given by (VRK-method)

$$
\left\{\begin{array}{r}
Y_{i}^{(n)}=\tilde{F}_{n}\left(x_{n}+\theta_{i} h\right)+h \sum_{j=1}^{m} a_{i j} K\left(x_{n}+d_{i j} h, x_{n}+c_{j} h, Y_{j}^{(n)}\right) \\
(i=1, \ldots, m), \\
y_{n+1}=Y_{m+1}^{(n)}=\tilde{F}_{n}\left(x_{n}+h\right)+h \sum_{i=1}^{m} b_{i} K\left(x_{n}+e_{i} h, x_{n}+c_{i} h, Y_{i}^{(n)}\right) .
\end{array}\right.
$$

We will always assume that

$$
c_{i}=\sum_{j=1}^{m} a_{i j} \quad(i=1, \ldots, m) .
$$

The method (1.3) is completely characterized by the parameters $a_{i j}, d_{i j}, b_{i}, e_{i}, \theta_{i}$. In the following we shall often refer to the two terms on the right-hand side of (1.3) as

Received September 29, 1980; revised October 21, 1981.

1980 Mathematics Subject Classification. Primary 65R20.

Key words and phrases. Volterra integral equations of the second kind, Runge-Kutta methods, order conditions. 
the "lag term" and the "Runge-Kutta part" of the Runge-Kutta method. Let us consider two special cases.

(A) Pouzet-Type Methods (PRK-Methods). If $d_{i j}=c_{i}(i, j=1, \ldots, m), e_{i}=1$, $\theta_{i}=c_{i}(i=1, \ldots, m)$, we obtain

$$
\left\{\begin{array}{r}
Y_{i}^{(n)}=\tilde{F}_{n}\left(x_{n}+c_{i} h\right)+h \sum_{j=1}^{m} a_{i j} K\left(x_{n}+c_{i} h, x_{n}+c_{j} h, Y_{j}^{(n)}\right) \\
\quad(i=1, \ldots m), \\
y_{n+1}=Y_{m+1}^{(n)}=\tilde{F}_{n}\left(x_{n}+h\right)+h \sum_{i=1}^{m} b_{i} K\left(x_{n}+h, x_{n}+c_{i} h, Y_{i}^{(n)}\right) .
\end{array}\right.
$$

This is the (implicit) version of Pouzet's Runge-Kutta method for (1.1) (compare Pouzet [14]); in the explicit case the upper limit of summation is replaced by $i-1$ in the first formula of (1.5). We observe that the "number" of kernel evaluations (per step) in the Runge-Kutta part is in general equal to $m(m+1)$ (implicit case), and $m(m+1) / 2$ (explicit case). This number is reduced if some of the parameters $a_{i j}$ vanish or if some of the $c_{i}$ 's are equal. In order that the argument of $K$ in (1.5) lies in $S \times R^{n}$, we have to demand that

$$
c_{i} \geqslant c_{j} \text { if } a_{i j} \neq 0
$$

For explicit methods this condition is satisfied if $c_{1} \leqslant c_{2} \leqslant \cdots \leqslant c_{m} \leqslant 1$. We shall refer to (1.6) as the kernel condition.

(B) Bel'tyukov-Type Methods (BRK-Methods). If $d_{i j}=e_{j}(i, j=1, \ldots, m), \theta_{i}=c_{i}$ $(i=1, \ldots, m)$, then

$$
\left\{\begin{aligned}
& Y_{i}^{(n)}=\tilde{F}_{n}\left(x_{n}+c_{i} h\right)+h \sum_{j=1}^{m} a_{i j} K\left(x_{n}+e_{j} h, x_{n}+c_{j} h, Y_{j}^{(n)}\right)(i=1, \ldots, m), \\
& y_{n+1}=Y_{m+1}^{(n)}=\tilde{F}_{n}\left(x_{n}+h\right)+h \sum_{i=1}^{m} b_{i} K\left(x_{n}+e_{i} h, x_{n}+c_{i} h, Y_{i}^{(n)}\right) .
\end{aligned}\right.
$$

This is the (implicit) Runge-Kutta method introduced by Bel'tyukov [3]; here, the "number" of kernel evaluations in the Runge-Kutta part equals $m$, independent of whether the method is implicit or explicit. For this type of methods the kernel condition reads as

$$
e_{i} \geqslant c_{i}, \quad i=1, \ldots, m .
$$

We remark that every method (1.3) (also the PRK-methods) can be written in the form (1.7) with a possible increase in $n$ (the number of stages).

The principal motivation for the present work originated with the following questions (whose answer will play a crucial role in connection with the selection of a computationally efficient VRK-method):

(i) If a Runge-Kutta method of order $p$ is given (i.e., the parameters $a_{i j}, b_{i}$ ), is then the corresponding Pouzet-type method (1.5) of the same order? This is proved in the explicit case for $p=m$ (see [14]), but is not yet clear for the general (implicit) case. 
(ii) If the first question is answered affirmatively, we obtain a large number of high order Pouzet-type methods. But, for a given order $p$, is it possible to reduce the number of kernel evaluations if we admit Bel'tyukov-type methods? For $p=3$ there exist explicit BRK-methods with $m=3$, whereas for PRK-methods at least four kernel evaluations are needed.

In order to deal with these problems (especially for high orders), we need a way of getting the order conditions for VRK-methods. In Brunner and Nørsett [4] these conditions were given by extending the Runge-Kutta theory of Butcher ([5], [6]) and of Hairer and Wanner ([7], [8]). However, at the same time Hairer [9] extended the theory in [7], [8] to what he called partitioned methods for partitioned systems of ordinary differential equations.

After transforming (1.1) to a canonical form, we may write (1.1) formally as an infinite system of ordinary differential equations. The difference between the solution of the " $M$ first" of these equations and the solution of (1.1) is of order $O\left(h^{M+1}\right)$ for $x \in\left[x_{0}, x_{0}+h\right]$. We can therefore also use that theory to find the Taylor expansion of the solution of (1.1) and in turn the order conditions for the VRKmethods. We will, in this paper, obtain our results in this way.

In Section 2 the theory of $V$-series will be presented and used to obtain the order conditions for the VRK-methods. The answer to question (i) is given in Section 3 together with a variety of examples of (explicit and implicit) Volterra-Runge-Kutta methods. Finally, Section 4 looks at some connections with other Runge-Kutta methods (Aparo [1], Ouelès [12], [13]).

2. Volterra Series and Order Conditions. As pointed out in Section 1, we will use the theory of $P$-series by Hairer [9] to derive the order conditions. It is therefore necessary to give a short review of the main results from that theory.

Consider the partitioned system of differential equations

$$
y_{a}^{\prime}=f_{a}\left(y_{a}, y_{b}, \ldots\right), \quad y_{b}^{\prime}=f_{b}\left(y_{a}, y_{b}, \ldots\right), \ldots,
$$

where $y_{a} \in R^{n_{a}}, \quad y_{b} \in R^{n_{b}}, \quad n=n_{a}+n_{b}+\ldots, \quad y=\left(y_{a}, y_{b}, \ldots\right)^{T}, f(y)=$ $\left(f_{a}(y), f_{b}(y), \ldots\right)^{T}$ and $A=\{a, b, \ldots\}$ is a finite index set. The function $f: U \rightarrow R^{n}$ is assumed to be infinitely differentiable, where $U$ is an open set in $R^{n}$.

The Taylor expansion of (2.1) is related to the concept of $P$-trees, defined by

Definition 2.1. A rooted $P$-tree $t$ of order $\rho(t)$ and root index $z=: w(t)$ is defined recursively as,

(i) $\phi_{z}, z \in A$ are the only $P$-trees of order 0 .

(ii) $\tau_{z}, z \in A$ are the only $P$-trees of order 1 .

(iii) Let $t_{1}, \ldots, t_{m}$ be $P$-trees with $\rho\left(t_{i}\right) \geqslant 1, z \in A$. Then $t={ }_{z}\left[t_{1}, \ldots, t_{m}\right]$ is a $P$-tree of order $\rho(t)=\sum_{i=1}^{m} \rho\left(t_{i}\right)+1$.

The ordering of the $P$-trees $t_{1}, \ldots, t_{m}$ in $t$ is irrelevant. $T P$ is the set of all $P$-trees.

Remark. Geometrically the $P$-trees can be represented by graphs as follows.

Order 1.

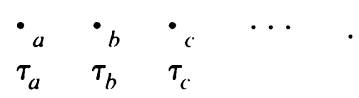


Order 2.

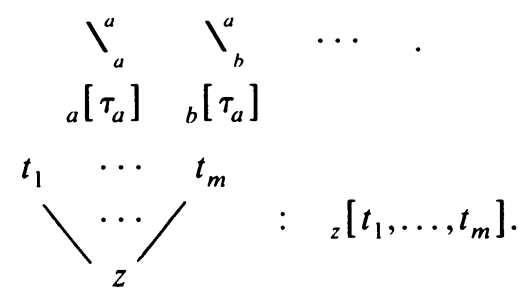

The node with index $z$ is called the root of $t$.

Hence $t$ is obtained by: The roots of $t_{1}, \ldots, t_{m}$ are connected by new arcs with a new node (with index $z$ ) which becomes the root of the new $P$-tree.

Definition. 2.2. For $t \in T P$ we define the integers $\alpha(t)$ recursively by,

(i) $\alpha\left(\phi_{z}\right)=\alpha\left(\tau_{z}\right)=1, z \in A$.

(ii) For $t={ }_{z}\left[t_{1}, \ldots, t_{m}\right], z \in A$,

$$
\alpha(t)=\left(\begin{array}{c}
\rho(t)-1 \\
\rho\left(t_{1}\right), \ldots, \rho\left(t_{m}\right)
\end{array}\right) \cdot \alpha\left(t_{1}\right) \cdot \cdots \cdot \alpha\left(t_{m}\right) \cdot \frac{1}{\mu_{1} ! \mu_{2} ! \ldots},
$$

where $\mu_{1}, \mu_{2}, \ldots$ are the numbers of mutually equal $P$-trees among $t_{1}, \ldots, t_{m}$.

Remark 2.3. This coefficient $\alpha(t)$ expresses the number of ways of monotonically labelling the nodes of $t$ with the numbers $1,2, \ldots, \rho(t)$ starting at the root.

Definition 2.4. For every $t \in T P$ we define a function $F(t): U \rightarrow R^{n}$ recursively by: Let $y=\left(y_{a}, y_{b}, \ldots\right)^{T} \in U$, then

(i) $F\left(\phi_{z}\right)(y)=y_{z}, z \in A$.

(ii) $F\left(\tau_{z}\right)(y)=f_{z}(y), z \in A$.

(iii) For $t={ }_{z}\left[t_{1}, \ldots, t_{m}\right], z \in A$

$$
F(t)(y)=\frac{\partial^{m} f_{z}(y)}{\partial y_{w\left(t_{1}\right)} \cdots \partial y_{w\left(t_{m}\right)}}\left(F\left(t_{1}\right)(y), \ldots, F\left(t_{m}\right)(y)\right) .
$$

The functions $F(t)(y)$ are called elementary differentials.

From Hairer [9].

THEOREM 2.5. For the solution of (2.1) we have

$$
y_{z}\left(x_{0}+h\right)=\sum_{t \in T P, w(t)=z} \alpha(t) F(t)\left(y_{0}\right) \frac{h^{\rho(t)}}{\rho(t) !}, \quad z \in A .
$$

Definition 2.6. Let $f: U \rightarrow R^{n}$ be as before and let $\Phi: T P \rightarrow R$. A P-series is a formal series of the form

$$
P(\Phi, y)=\left(P_{z}(\Phi, y)\right)_{z \in A}=\left(\sum_{t \in T P, w(t)=z} \Phi(t) \alpha(t) F(t)(y) \frac{h^{\rho(t)}}{\rho(t) !}\right)_{z \in A} .
$$

Theorem 2.7. Let $P(\Phi, y)$ be a $\dot{P}$-series with $\Phi\left(\phi_{z}\right)=1, z \in A$. Then $h f(P(\Phi, y))$ is formally a $P$-series $P\left(\Phi^{\prime}, y\right)$, where

$$
\begin{gathered}
\Phi^{\prime}\left(\phi_{z}\right)=0, \quad z \in A, \\
\Phi^{\prime}\left(\tau_{z}\right)=1, \quad z \in A, \\
\Phi^{\prime}(t)=\rho(t) \Phi\left(t_{1}\right) \cdots \Phi\left(t_{m}\right), \quad t={ }_{z}\left[t_{1}, \ldots, t_{m}\right], \quad z \in A .
\end{gathered}
$$


Instead of Eq. (1.1) we now consider, without loss of generality in the subsequent sections (recall (1.4), and compare also Section 4), the canonical Volterra equation

$$
y(x)=\int_{x_{0}}^{x} G(x, y(s)) d s, \quad x \in I,
$$

assuming $G$ to be sufficiently smooth.

In order to use the theory of $P$-series, we have to write $(2.2)$ as a system of differential equations. For that purpose we set

$$
A=\left\{a_{i} ; i=0,1,2, \ldots\right\} \cup\{x\}, \quad a=a_{0},
$$

and further

$$
\left\{\begin{array}{l}
y_{a}(x)=y(x) \\
y_{a_{i}}(x)=\int_{x_{0}}^{x} \frac{\partial^{i}}{\partial x^{i}} G\left(x, y_{a}(s)\right) d s, \quad i=0,1, \ldots \\
y_{x}(x)=x
\end{array}\right.
$$

Then

$$
\left\{\begin{array}{l}
y_{a_{i}}^{\prime}=\frac{\partial^{i}}{\partial x^{i}} G\left(y_{x}, y_{a}\right)+y_{a_{i+1}}, \quad i=0,1, \ldots, ; \quad y_{a_{i}}\left(x_{0}\right)=0, \\
y_{x}^{\prime}=1 ; \quad y_{x}\left(x_{0}\right)=x_{0} .
\end{array}\right.
$$

Now

$$
\begin{aligned}
& y^{\prime}(x)=y_{a}^{\prime}(x)=G\left(y_{x}, y_{a}\right)+y_{a_{1}}, \\
& y^{\prime \prime}(x)=y_{a}^{\prime \prime}(x)=G_{x}+G_{y} \cdot y_{a}^{\prime}+y_{a_{1}}^{\prime}=G_{x}+G_{y} \cdot y_{a}^{\prime}+G_{x}+y_{a_{2}},
\end{aligned}
$$

and we see that $y^{(k)}(x)$ only depends on $y_{x}, y_{a_{i}}, i=0, \ldots, k$. Thus, for the computation of the truncated Taylor expansion of $y(x)$ we may assume that $A$ is finite as far as we need.

Furthermore, our system (2.5) is very special in its structure. From Theorem 2.5 we immediately get

$$
y_{a}\left(x_{0}+h\right)=\sum_{t \in T P, w(t)=a} \alpha(t) F(t)\left(y_{0}\right) \frac{h^{\rho(t)}}{\rho(t) !},
$$

with $y_{0}=\left(0,0, \ldots, 0, x_{0}\right)$. But, due to the structure of $(2.5)$, two facts have to be taken into consideration.

First, for the system (2.5) a lot of elementary differentials in (2.6) vanish. For example,

$$
\begin{gathered}
F\left(a_{0}\left[\tau_{a_{2}}\right]\right)(y)=\frac{\partial f_{a_{0}}}{\partial y_{a_{2}}} \cdot f_{a_{2}}=0, \\
F\left({ }_{a_{0}}\left[\tau_{x}, \tau_{a_{1}}\right]\right)(y)=\frac{\partial^{2} f_{a_{0}}}{\partial y_{x} \cdot \partial y_{a_{1}}} \cdot\left(f_{x}, f_{a_{1}}\right)=0 .
\end{gathered}
$$

Secondly, and this has not been seen for a general system of ordinary differential equations, some of the nonvanishing elementary differentials are equal. For example,

$$
F\left(a_{0}\left[\tau_{x}\right]\right)\left(y_{0}\right)=\frac{\partial f_{a_{0}}}{\partial y_{x}} \cdot f_{x}=G_{x}
$$


and

$$
F\left(a_{0}\left[\tau_{a_{1}}\right]\right)\left(y_{0}\right)=\left.\frac{\partial f_{a_{0}}}{\partial y_{a_{1}}} \cdot f_{a_{1}}\right|_{y_{0}}=G_{x} .
$$

Hence only a subset of $T P$ is relevant for (2.6) or for the Taylor expansion of the solution $y$ of (2.2).

Definition 2.8. With $T V$ (Volterra-trees) we denote the smallest subset of $T P$ satisfying

(i) $\phi_{a} \in T V, \tau_{a} \in T V$,

(ii) If $t_{1}, \ldots, t_{m} \in T V, \rho\left(t_{i}\right) \geqslant 1$, then

$$
t={ }_{a}[\underbrace{\tau_{x}, \tau_{x}, \ldots, \tau_{x}}_{k}, t_{1}, \ldots, t_{m}]=:{ }_{a}\left[\tau_{x}^{k}, t_{1}, \ldots, t_{m}\right]
$$

is also in $T V$.

(In this definition the cases $m=0$ and $k=0$ are included. For $m=k=0,{ }_{a}[]$ is to be interpreted as $\tau_{a}$.)

The elements of $T V$ are exactly those $P$-trees which are indexed only by " $a$ " and " $x$ ", and if a node has index " $x$ ", this node must be an end-node. $\tau_{x}$ is not in $T V$. This set $T V$ also corresponds to the set of Volterra-trees of Brunner and Norsett [4]. There the numbers at the nodes correspond to the free $x$-nodes leaving that node.

Example.

$$
\begin{aligned}
& \rho(t)=0 \quad: \phi_{a} \\
& \rho(t)=1 \quad: \dot{a}^{a} \\
& \rho(t)=2: \backslash_{a}^{x} \backslash_{a}^{a} \\
& \rho(t)=3: \searrow_{a} \nearrow^{x} \searrow_{a} \nearrow^{a} \searrow_{a} \nearrow_{a}^{a}>_{a}{ }_{a}^{a}>a
\end{aligned}
$$

Having defined the set $T V$ of trees, we need to find which trees in $T P$ give $F(t)\left(y_{0}\right)=0$ and which give the same results as trees in $T V$. In this connection we set

Definition 2.9. For every $t \in T V$ we define $E(t) \subset T P$ recursively by:

(i) $E\left(\phi_{a}\right)=\left\{\phi_{a}\right\}, E\left(\tau_{a}\right)=\left\{\tau_{a}\right\}$.

(ii) If $t={ }_{a}\left[\tau_{x}^{k}, t_{1}, \ldots, t_{m}\right], t_{i} \in T V, \rho\left(t_{i}\right) \geqslant 1$, then

$$
E(t)=\bigcup_{i=0}^{k} E_{i}(t)
$$

where

$$
E_{j}(t)=\left\{a_{0}\left[a_{1}\left[\cdots a_{j}\left[\tau_{x}^{k-j}, u_{1}, \ldots, u_{m}\right] \cdots\right]\right] ; u_{i} \in E\left(t_{i}\right)\right\}, \quad j=0,1,2, \ldots, k .
$$

Example 2.10. For $t={ }_{a}\left[\tau_{x}, \tau_{x}\right]$,

$$
E(t)=\left\{a_{0}\left[\tau_{x}, \tau_{x}\right],{ }_{a_{0}}\left[a_{1}\left[\tau_{x}\right]\right],{ }_{a_{0}}\left[{ }_{a_{1}}\left[a_{2}[]\right]\right]={ }_{a_{0}}\left[a_{1}\left[\tau_{a_{2}}\right]\right]\right\}
$$


Based on this definition we have

THEOREM 2.11. The elementary differentials corresponding to (2.5) have the following properties:

(i) $u \in E(t) \Rightarrow F(u)\left(y_{0}\right)=F(t)\left(y_{0}\right)$;

(ii) $u \notin \cup_{t \in T V} E(t)$ and $w(u)=a \Rightarrow F(u)\left(y_{0}\right)=0$.

Proof. The first statement is proved by induction on the order of $t$. Let now $u \in T P$ with $w(u)=a$. From the definition of $f_{a}$ it follows that $F(u)\left(y_{0}\right)=0$ except when $u$ has either the form ${ }_{a}\left[\tau_{x}^{k}, u_{1}, \ldots, u_{m}\right]$ with $w\left(u_{i}\right)=a$ or ${ }_{a}\left[u_{1}\right]$ with $w\left(u_{1}\right)=a_{1}$. In the first case the statement follows by an induction argument. In the second case the definition of $f_{a_{1}}$ implies that $F(u)\left(y_{0}\right)=0$ except when $u_{1}$ has either the form ${ }_{a_{1}}\left[\tau_{x}^{k-1}, v_{1}, \ldots, v_{m}\right]$ with $w\left(v_{i}\right)=a$ or $u_{1}={ }_{a_{1}}\left[v_{1}\right]$ with $w\left(v_{1}\right)=a_{2}, \ldots$ etc.

Combining these results, we have

THEOREM 2.12. For the solution of (2.2) we have

$$
y\left(x_{0}+h\right)=\sum_{t \in T V} \beta(t) F(t)\left(y_{0}\right) \frac{h^{\rho(t)}}{\rho(t) !},
$$

where

$$
\beta(t)=\sum_{u \in E(t)} \alpha(u)
$$

By using Definition 2.2, (ii) and Definition 2.9, (ii) we get $\left(t={ }_{a}\left[\tau_{x}^{k}, t_{1}, \ldots, t_{m}\right]\right)$

$$
\begin{aligned}
& \beta(t)=\sum_{i=0}^{k} \sum_{u \in E_{i}(t)} \alpha(u) \\
& =\sum_{i=0}^{k} \sum_{u_{1} \in E\left(t_{1}\right)} \ldots \sum_{u_{m} \in E\left(t_{m}\right)} \frac{(\rho(t)-i-1) !}{\rho\left(t_{1}\right) ! \ldots \rho\left(t_{m}\right) !} \cdot \alpha\left(u_{1}\right) \cdots \alpha\left(u_{m}\right) \frac{1}{(k-i) ! \mu_{1} ! \mu_{2} ! \ldots} \\
& =\sum_{i=0}^{k} \frac{(\rho(t)-i-1) !}{\rho\left(t_{1}\right) ! \ldots \rho\left(t_{m}\right) !} \cdot \beta\left(t_{1}\right) \cdots \beta\left(t_{m}\right) \cdot \frac{1}{(k-i) ! \mu_{1} ! \mu_{2} ! \ldots},
\end{aligned}
$$

where $\mu_{1}, \mu_{2}, \ldots$ are the numbers of mutually equal $P$-trees among $t_{1}, \ldots, t_{m}$. Since

$$
\begin{aligned}
\sum_{i=0}^{k} \frac{(\rho(t)-i-1) !}{(k-i) !} & =(\rho(t)-k-1) ! \sum_{i=0}^{k}\left(\begin{array}{c}
(\rho(t)-i-1) \\
k-i
\end{array}\right) \\
& =(\rho(t)-k-1) !\left(\begin{array}{c}
\rho(t) \\
k
\end{array}\right)=\frac{\rho(t) !}{k !(\rho(t)-k)},
\end{aligned}
$$

we finally get for the recursive calculation of $\beta(t)$,

$$
\begin{aligned}
\beta(t)= & \frac{\rho(t)}{(\rho(t)-k)}\left(\begin{array}{c}
\rho(t)-1 \\
\underbrace{1, \ldots, 1}_{k}, \rho\left(t_{1}\right), \ldots, \rho\left(t_{m}\right)
\end{array}\right) \\
& \cdot \beta\left(t_{1}\right) \cdots \beta\left(t_{m}\right) \cdot \frac{1}{k ! \mu_{1} ! \mu_{2} ! \ldots}
\end{aligned}
$$


Example 2.13.

\begin{tabular}{|c|c|c|c|c|}
\hline & $t$ & $\alpha(t)$ & $\beta(t)$ & $F(t)\left(y_{0}\right)$ \\
\hline$\rho(t)=1$ & & 1 & 1 & $G$ \\
\hline \multirow[t]{2}{*}{$\rho(t)=2$} & & 1 & 2 & $G_{x}$ \\
\hline & & 1 & 1 & $G_{y} G$ \\
\hline \multirow[t]{5}{*}{$\rho(t)=3$} & & 1 & 3 & $G_{x x}$ \\
\hline & & 2 & 3 & $G_{x y} G$ \\
\hline & & 1 & 1 & $G_{y y} G G$ \\
\hline & & 1 & 2 & $G_{y} G_{x}$ \\
\hline & & 1 & 1 & $G_{y} G_{y} G$ \\
\hline
\end{tabular}

Hence from (2.7),

$$
\begin{aligned}
y(x)= & G \cdot h+\left(2 G_{x}+G_{y} G\right) \cdot \frac{h^{2}}{2} \\
& +\left(3 G_{x x}+3 G_{x y} G+G_{y y} G G+2 G_{y} G_{x}+G_{y} G_{y} G\right) \cdot \frac{h^{3}}{6}+O\left(h^{4}\right) .
\end{aligned}
$$

The VRK-method for (2.2) takes the form

$$
\left\{\begin{array}{l}
Y_{i}=h \sum_{j=1}^{m} a_{i j} G\left(x_{0}+d_{i j} h, Y_{j}\right), \quad i=1, \ldots, m, \\
y_{1}=h \sum_{i=1}^{m} b_{i} G\left(x_{0}+e_{i} h, Y_{i}\right) .
\end{array}\right.
$$

From Theorem 2.12 the exact solution has an expansion in terms of Volterra-trees. It would therefore be natural to expect $y_{1}$ also to have an expansion of that form except that $\beta(t)$ in (2.7) would be other coefficients. Analogously to Definition 2.6,

Definition 2.14. Let $G$ be smooth enough and let $\varphi: T V \rightarrow R$. A $V$-series is a formal series of the form

$$
V(\varphi, y)=\sum_{t \in T V} \varphi(t) \beta(t) F(t)(y) \frac{h^{\rho(t)}}{\rho(t) !} .
$$

We now need a result of the form $h G\left(x_{0}+d h, V(\varphi, y)\right)=V\left(\varphi^{\prime}(d, \cdot), y\right)$. For the general case this was given by Theorem 2.7 .

THEOREM 2.15. Let $\varphi: T V \rightarrow R, \varphi\left(\phi_{a}\right)=1$. Then

$$
h G\left(x_{0}+d h, V\left(\varphi, y_{0}\right)\right)=V\left(\varphi^{\prime}(d, \cdot), y_{0}\right)
$$

where

$$
\begin{gathered}
\varphi^{\prime}\left(d, \phi_{a}\right)=0, \quad \varphi^{\prime}\left(d, \tau_{a}\right)=1, \\
\varphi^{\prime}(d, t)=(\rho(t)-k) d^{k} \varphi\left(t_{1}\right) \cdot \cdots \cdot \varphi\left(t_{m}\right) \quad \text { for } t={ }_{a}\left[\tau_{x}^{k}, t_{1}, \ldots, t_{m}\right] .
\end{gathered}
$$


Proof. Let

$$
\Phi(t):= \begin{cases}\varphi(t) \cdot \frac{\beta(t)}{\alpha(t)} & \text { for } t \in T V \\ d & \text { for } t=\tau_{x} \\ 1 & \text { if } \rho(t)=0 \\ 0 & \text { otherwise. }\end{cases}
$$

Then we have

$$
\begin{aligned}
& P_{a}\left(\Phi, y_{0}\right)=V\left(\varphi, y_{0}\right) \\
& P_{x}\left(\Phi, y_{0}\right)=x_{0}+d h \\
& P_{a_{i}}\left(\Phi, y_{0}\right)=0 \text { for } i=1,2, \ldots
\end{aligned}
$$

This and Theorem 2.7 imply

$$
\begin{aligned}
h G\left(x_{0}+d h, V\left(\varphi, y_{0}\right)\right) & =h f_{a}\left(P\left(\Phi, y_{0}\right)\right)=P_{a}\left(\Phi^{\prime}, y_{0}\right) \\
& =\sum_{t \in T V} \Phi^{\prime}(t) \alpha(t) F(t)\left(y_{0}\right) \frac{h^{\rho(t)}}{\rho(t) !} .
\end{aligned}
$$

The last equality holds since $\Phi^{\prime}(t)=0$ for all $P$-trees $t$ which have root index " $a$ " but do not belong to $T V$. Putting

$$
\varphi^{\prime}(d, t):=\Phi^{\prime}(t) \cdot \frac{\alpha(t)}{\beta(t)} \quad \text { for } t \in T V,
$$

we thus have $h G\left(x_{0}+d h, V\left(\varphi, y_{0}\right)\right)=V\left(\varphi^{\prime}(d, \cdot), y_{0}\right)$. The recurrence relation for $\varphi^{\prime}(d, \cdot)$ follows from those of $\Phi^{\prime}, \alpha$ and $\beta$ (Theorem 2.7, Definition 2.2 and formula (2.9)).

We are now able to prove that the numerical solution $y_{1}$ given by $(2.10)$ is a $V$-series.

THEOREM 2.16. If the kernel $G$ is sufficiently smooth, then the numerical solutions $y_{1}$ and $Y_{i}(i=1, \ldots, m)$ given by (2.10), are $V$-series

$$
y_{1}=V\left(\varphi, y_{0}\right), \quad Y_{i}=V\left(\varphi_{i}, y_{0}\right) .
$$

The coefficients are given by

$$
\left\{\begin{array}{l}
\varphi_{i}\left(\phi_{a}\right)=0, \quad \varphi\left(\phi_{a}\right)=0, \\
\varphi_{i}\left(\tau_{a}\right)=c_{i}, \quad \varphi\left(\tau_{a}\right)=\sum_{i=1}^{m} b_{i}, \\
\varphi_{i}(t)=(\rho(t)-k) \sum_{j=1}^{m} a_{i j} d_{i j}^{k} \varphi_{j}\left(t_{1}\right) \cdots \cdots \varphi_{j}\left(t_{q}\right), \\
\varphi(t)=(\rho(t)-k) \sum_{i=1}^{m} b_{i} e_{i}^{k} \varphi_{i}\left(t_{1}\right) \cdots \cdots \varphi_{i}\left(t_{q}\right) \quad \text { if } t={ }_{a}\left[\tau_{x}^{k}, t_{1}, \ldots, t_{q}\right] .
\end{array}\right.
$$

Proof. Inserting the assumption (2.13) into (2.10), we obtain by comparing the coefficients

$$
\varphi_{i}(t)=\sum_{j=1}^{m} a_{i j} \varphi_{j}^{\prime}\left(d_{i j}, t\right), \quad \varphi(t)=\sum_{i=1}^{m} b_{i} \varphi_{i}^{\prime}\left(e_{i}, t\right) .
$$


Formula (2.14) now follows from (2.12). The validity of the assumption (2.13) is trivial if the RVK-method is explicit and is a consequence of the implicit function theorem in the general case.

The following result is now obvious.

THEOREM 2.17. Let $\varphi: T V \rightarrow R$ be given by (2.14). Then the local truncation error of the VRK-method (2.10) is given by

$$
y_{1}-y\left(x_{1}\right)=\sum_{t \in T V}(\varphi(t)-1) \beta(t) F(t)\left(y_{0}\right) \frac{h^{\rho(t)}}{\rho(t) !},
$$

and the VRK-method has order $p$ if

$$
\varphi(t)=1 \text { for } \rho(t) \leqslant p, t \in T V .
$$

We conclude this section by an example. For the $V$-tree in the following figure the condition $(2.15)$ is given by

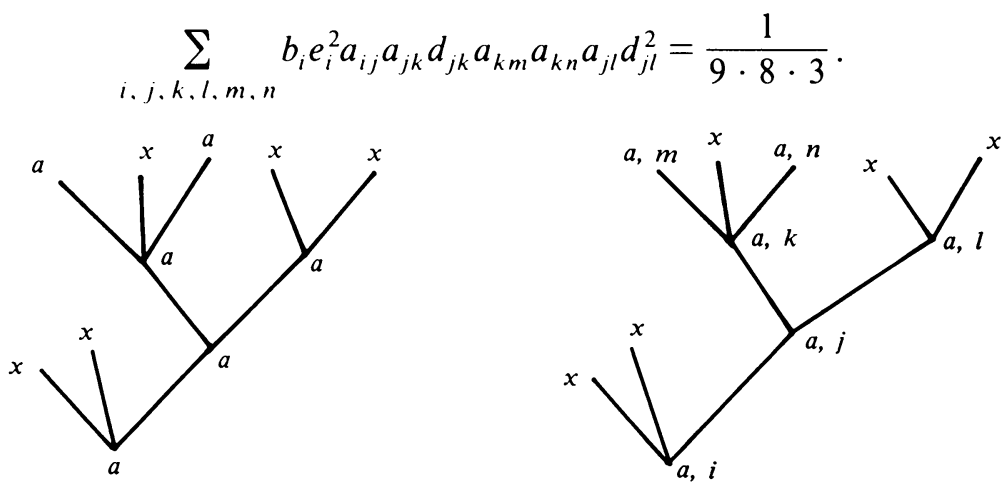

This condition can be obtained very elegantly. If we affix to every node with index " $a$ " a summation index $i, j, k, l, \ldots$, then the left-hand side of (2.16) is obtained as the sum over $i, j, k, l, \ldots$, whose summand is a product of

$b_{i} e_{i}^{k}$ if the summation index of the root is $i$ and if the root is connected with $k$ nodes " $x$ ";

$a_{i j} d_{i j}^{k}$ if a lower node (with summation index $i$ ) is connected with a higher node " $j$ ", and if this higher node is directly connected with $k$ nodes " $x$ ".

The right-hand side is the inverse of $\gamma(t)$, where $\gamma(t)$ is defined for $t \in T V$ as

$$
\begin{gathered}
\gamma\left(\phi_{a}\right)=\gamma\left(\tau_{a}\right)=1, \\
\gamma(t)=(\rho(t)-k) \gamma\left(t_{1}\right) \cdot \cdots \cdot \gamma\left(t_{m}\right) \text { for } t={ }_{a}\left[\tau_{x}^{k}, t_{1}, \ldots, t_{m}\right] .
\end{gathered}
$$

3. Examples of Volterra-Runge-Kutta Methods. In Section 1 we defined the general VRK-method. As particular subclasses we had the Pouzet-methods and the Bel'tyukov-methods. Pouzet [14] showed that for every given explicit $m$-stage $R K$ method of order $p=m$ for ordinary differential equations the corresponding Pouzet-method also had order $p$. (The converse is obviously true.) By using the theory of $V$-series we can in general establish

THEOREM 3.1. Let $a_{i j}(i, j=1, \ldots, m)$ and $b_{i}(i=1, \ldots, m)$ represent a $R K$-method of order $p$. Then the corresponding Pouzet-method has order $p$. 
Proof. Let $T=\{t \in T V$; all nodes of $t$ have index " $a$ " $\}$. By assumption the $R K$-method has order $p$. Since for $t \in T$ the order condition (2.15) is exactly the same as for $R K$-methods (see [6]) we have

$$
\varphi(t)=1 \text { for } \rho(t) \leqslant p, t \in T .
$$

With $R(t)$ (for $t \in T V$ ) we denote the number of nodes indexed by " $x$ " which are directly connected with the root of $t$. For an arbitrary element $t \in T V$ we then define $u(t) \in T$ recursively by

$$
\begin{gathered}
u\left(\phi_{a}\right)=\phi_{a}, \quad u\left(\tau_{a}\right)=\tau_{a}, \\
u(t)={ }_{a}\left[\tau_{a}^{R\left(t_{1}\right)+\cdots+R\left(t_{m}\right)}, u\left(t_{1}\right), \ldots, u\left(t_{m}\right)\right] \text { for } t={ }_{a}\left[\tau_{x}^{R(t)}, t_{1}, \ldots, t_{m}\right] .
\end{gathered}
$$

Observe that $u(t) \in T$ and $\rho(u(t))=\rho(t)-R(t)$. An easy induction argument using the formulas (2.14) with $d_{i j}=c_{i}$ and $e_{i}=1$ shows that

$$
\varphi_{i}(t)=c_{i}^{R(t)} \varphi_{i}(u(t)) \text { and } \varphi(t)=\varphi(u(t)) .
$$

This last relation together with (3.1) completes the proof.

In the following examples the methods will be given for the problem

$$
y(x)=f(x)+\int_{x_{0}}^{x} K(x, s, y(s)) d s .
$$

Example 3.2. $m=1$.

$$
\left.\begin{array}{l}
\operatorname{order} 1: b_{1}=1 \\
\operatorname{order} 2: b_{1} e_{1}=1, b_{1} c_{1}=1 / 2
\end{array}\right\} \Rightarrow b_{1}=1, c_{1}=1 / 2, e_{1}=1 .
$$

With $c_{1}=0$ the explicit methods of order 1 are

$$
\left\{\begin{array}{l}
Y_{1}=f\left(x_{0}\right) \\
y_{1}=f\left(x_{0}+h\right)+h K\left(x_{0}+e_{1} h, x_{0}, Y_{1}\right) .
\end{array}\right.
$$

The methods of order 2 will be

$$
\left\{\begin{array}{l}
Y_{1}=f\left(x_{0}+h / 2\right)+\frac{h}{2} \cdot K\left(x_{0}+d_{11} h, x_{0}+h / 2, Y_{1}\right), \\
y_{1}=f\left(x_{0}+h\right)+h K\left(x_{0}+h, x_{0}+h / 2, Y_{1}\right) .
\end{array}\right.
$$

For $d_{11}=1\left(=e_{1}\right)$ we obtain the Bel'tyukov-type midpoint method,

$$
\left\{\begin{array}{l}
Y_{1}=f\left(x_{0}+h / 2\right)+\frac{h}{2} \cdot K\left(x_{0}+h, x_{0}+h / 2, Y_{1}\right), \\
y_{1}=f\left(x_{0}+h\right)+h K\left(x_{0}+h, x_{0}+h / 2, Y_{1}\right),
\end{array}\right.
$$

while for the choice $d_{11}=1 / 2$, we have the Pouzet-type midpoint method,

$$
\left\{\begin{array}{l}
Y_{1}=f\left(x_{0}+h / 2\right)+\frac{h}{2} \cdot K\left(x_{0}+h / 2, x_{0}+h / 2, Y_{1}\right), \\
y_{1}=f\left(x_{0}+h\right)+h K\left(x_{0}+h, x_{0}+h / 2, Y_{1}\right) .
\end{array}\right.
$$

Note that $\left(3.3^{\prime}\right)$ requires only one kernel evaluation per step in the Runge-Kutta part but has order $2 ;\left(3.3^{\prime \prime}\right)$ requires two kernel evaluations. 
Example 3.3. Explicit two-stage VRK-methods.

$$
\begin{array}{cll}
\operatorname{order} 1: & { }_{a} & b_{1}+b_{2}=1, \\
\operatorname{order} 2: & \int_{a}^{x} & b_{1} e_{1}+b_{2} e_{2}=1, \\
& \int_{a}^{a} & b_{2} c_{2}=1 / 2 .
\end{array}
$$

Hence,

$$
b_{2}=1 /\left(2 c_{2}\right), \quad b_{1}=1-1 /\left(2 c_{2}\right), \quad e_{2}=2 c_{2}+\left(1-2 c_{2}\right) e_{1} .
$$

A particular example is given by choosing $c_{2}=2 / 3, e_{1}=1, d_{21}=1$, thus $b_{1}=1 / 4$, $b_{2}=3 / 4, e_{2}=1$, and we have a Bel'tyukov method of order two:

$$
\begin{aligned}
& Y_{1}=f\left(x_{0}\right), \\
& Y_{2}=f\left(x_{0}+2 h / 3\right)+\frac{2 h}{3} \cdot K\left(x_{0}+h, x_{0}, Y_{1}\right), \\
& y_{1}=f\left(x_{0}+h\right)+\frac{h}{4} \cdot\left\{K\left(x_{0}+h, x_{0}, Y_{1}\right)+3 K\left(x_{0}+h, x_{0}+2 h / 3, Y_{2}\right)\right\} ;
\end{aligned}
$$

i.e., we obtain a method listed on p. 420 of [3], where the number of kernel evaluations equals two. The Pouzet counterpart has the form

$$
\begin{aligned}
& Y_{1}=f\left(x_{0}\right), \\
& Y_{2}=f\left(x_{0}+2 h / 3\right)+\frac{2 h}{3} \cdot K\left(x_{0}+2 h / 3, x_{0}, Y_{1}\right), \\
& y_{1}=f\left(x_{0}+h\right)+\frac{h}{4} \cdot\left\{K\left(x_{0}+h, x_{0}, Y_{1}\right)+3 K\left(x_{0}+h, x_{0}+2 h / 3, Y_{2}\right)\right\} ;
\end{aligned}
$$

it uses three kernel evaluations per step.

We now turn our attention to Bel'tyukov methods. The order conditions for an $m$-stage BRK-method are obtained in the same way as formula (2.16) using $d_{i j}=e_{j}$.

order 1: (i) $\quad \sum_{i=1}^{m} b_{i}=1$;

order 2: (ii) $\sum_{i=1}^{m} b_{i} e_{i}=1$,

(iii) $\sum_{i=1}^{m} b_{i} c_{i}=1 / 2$;

order 3: (iv) $\sum_{i=1}^{m} b_{i} e_{i}^{2}=1$,

(v) $\sum_{i=1}^{m} b_{i} e_{i} c_{i}=\$ / 2$,

(vi) $\sum_{i=1}^{m} b_{i} c_{i}^{2}=1 / 3$,

(vii) $\sum_{i=1}^{m} b_{i} \sum_{j=1}^{m} a_{i j} e_{j}=1 / 3$,

(viii) $\sum_{i=1}^{m} b_{i} \sum_{j=1}^{m} a_{i j} c_{j}=1 / 6$. 
LEMMA 3.4. If a BRK-method has order $p \geqslant 3$, then at least one of the $e_{i}$-values is different from 1 .

Proof. Suppose that the order is greater than or equal to 3 and that $e_{i}=1$ for all $i$. The order conditions (iii) and (vii) then give a contradiction by (1.4).

THEOREM 3.5. There is no 2-stage BRK-method of order 3.

Proof. Suppose that $m=2$ and $p=3$. The conditions (i), (iii), and (vi) indicate that the underlying quadrature formula has order 3. Since no 3rd order quadrature formula exists with only one function evaluation, we have

$$
b_{1} \neq 0, \quad b_{2} \neq 0, \text { and } c_{1} \neq c_{2} \text {. }
$$

Subtracting the condition (i) from (ii) and (iii) from (v) we obtain

$$
\begin{aligned}
b_{1}\left(e_{1}-1\right)+b_{2}\left(e_{2}-1\right) & =0, \\
b_{1} c_{1}\left(e_{1}-1\right)+b_{2} c_{2}\left(e_{2}-1\right) & =0 .
\end{aligned}
$$

Hence we get $e_{1}=e_{2}=1$, but this is impossible by Lemma 3.4.

LEMMA 3.6. If a 3-stage BRK-method has order 3, then

$$
b_{i}\left(e_{i}-1\right)=0, \quad i=1,2,3 .
$$

Proof. By subtracting (i) and (ii), (iii) and (v), (ii) and (iv), we get

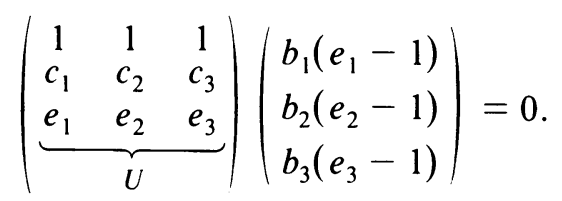

Suppose that there exists $\alpha=\left(\alpha_{1}, \alpha_{2}, \alpha_{3}\right)^{T} \neq 0$ such that $\alpha^{T} U=0$, i.e.,

$$
\alpha_{1}+\alpha_{2} c_{i}+\alpha_{3} e_{i}=0, \quad i=1,2,3 .
$$

If we multiply this equation with $b_{i}$ and take the sum over $i$, we obtain by (i), (ii), and (iii)

$$
\alpha_{1}+\alpha_{2} / 2+\alpha_{3}=0
$$

If we multiply the above equation with $b_{i} c_{i}$, we get in a similar way

$$
\alpha_{1} / 2+\alpha_{2} / 3+\alpha_{3} / 2=0
$$

The last two equations imply $\alpha_{2}=0$ and $\alpha_{1}+\alpha_{3}=0$, so that

$$
\alpha_{3}\left(e_{i}-1\right)=0 \text { for all } i \text {. }
$$

This contradicts $\alpha \neq 0$ by Lemma 3.4. Hence $\operatorname{det}(U) \neq 0$.

Since we need $b_{3} \neq 0$ for an explicit 3-stage RK-method to be of order 3, Lemma 3.6 implies $e_{3}=1 . b_{1}$ and $b_{2}$ cannot both be zero by (i), (iii), and (vi). By Lemma 3.6 we then have two cases, $b_{2}=0, e_{1}=e_{3}=1$ and $b_{1}=0, e_{2}=e_{3}=1$.

Case A: $b_{2}=0, e_{1}=e_{3}=1$. From (v) and (vi) $c_{3}=2 / 3, b_{3}=3 / 4$ implying $b_{1}=1 / 4$; from (vii), $a_{32}\left(1-e_{2}\right)=2 / 9$, while (viii) implies $c_{2}=1-e_{2}$;

$$
a_{32}=2 /\left(9\left(1-e_{2}\right)\right), \quad a_{31}=2\left(2-3 e_{2}\right) /\left(9\left(1-e_{2}\right)\right) \text {. }
$$


The kernel condition (1.8) is satisfied for $e_{2} \geqslant 1 / 2$. The corresponding method is therefore

$$
\left\{\begin{aligned}
k_{1}= & h K\left(x_{0}+h, x_{0}, f\left(x_{0}\right)\right) \\
k_{2}= & h K\left(x_{0}+e_{2} h, x_{0}+\left(1-e_{2}\right) h, f\left(x_{0}+\left(1-e_{2}\right) h\right)+\left(1-e_{2}\right) k_{1}\right) \\
k_{3}=h K\left(x_{0}+h, x_{0}+\right. & 2 h / 3, f\left(x_{0}+2 h / 3\right) \\
& \left.\quad+\left[2\left(2-3 e_{2}\right) k_{1}+2 k_{2}\right] /\left(9\left(1-e_{2}\right)\right)\right) \\
y_{1}= & f\left(x_{0}+h\right)+\left(k_{1}+3 k_{3}\right) / 4
\end{aligned}\right.
$$

In particular,

Example 3.7. 3-stage explicit Bel'tyukov method of order 3 (see also [3, p. 421]), $e_{2}=1 / 2$,

\begin{tabular}{c|c|ccc}
$e$ & $c$ & $A$ & & \\
\hline 1 & 0 & 0 & & \\
$1 / 2$ & $1 / 2$ & $1 / 2$ & 0 & \\
1 & $2 / 3$ & $2 / 9$ & $4 / 9$ & 0 \\
\hline & & $1 / 4$ & 0 & $3 / 4$
\end{tabular}

Case B: $b_{1}=0, e_{2}=e_{3}=1$. In this case the solution of the order conditions is given by

$$
\begin{gathered}
c_{1}=0, \quad c_{2}=\left(1-e_{1}\right) /\left(2-3 e_{1}\right), \quad c_{3}=1-1 /\left(3 e_{1}\right), \\
b_{2}=\left(2-3 e_{1}\right)^{2} /\left(4\left(1-3 e_{1}+3 e_{1}^{2}\right)\right), \quad b_{3}=1-b_{2}, \quad a_{21}=c_{2}, \\
a_{32}=\left(2-3 e_{1}\right) /\left(6\left(1-e_{1}\right)\left(1-b_{2}\right)\right), \quad a_{31}=c_{3}-a_{32},
\end{gathered}
$$

with $e_{1}$ as free parameter $\left(e_{1} \neq 0, e_{1} \neq 2 / 3, e_{1} \neq 1\right)$. For $e_{1} \leqslant 1 / 2$ the kernel condition (1.8) is satisfied. In particular, the choice $e_{1}=1 / 3$ yields method (18) of [3]:

\begin{tabular}{c|c|ccc}
$1 / 3$ & 0 & 0 & & \\
1 & $2 / 3$ & $2 / 3$ & 0 & \\
1 & 0 & -1 & 1 & 0 \\
\hline & & 0 & $3 / 4$ & $1 / 4$
\end{tabular}

THEOREM 3.8. There is no 4-stage explicit BRK-method of order 4.

Proof. Every 4-stage explicit RK-method of order 4 satisfies (see [6, p. 78])

$$
\sum_{i=1}^{4} b_{i} a_{i j}=b_{j}\left(1-c_{j}\right), \quad j=1,2,3,4 \text {. }
$$

If we multiply each side of this equation by $e_{j}$ and sum over $j$, we obtain

$$
\sum_{i=1}^{4} b_{i} \sum_{j=1}^{4} a_{i j} e_{j}=\sum_{j=1}^{4} b_{j} e_{j}-\sum_{j=1}^{4} b_{j} c_{j} e_{j}
$$

The order conditions (vii), (ii), and (v) imply that this equation is the same as $1 / 3=1-1 / 2$, which is a contradiction. 
Example 3.9. The following coefficients represent a 5-stage explicit BRK-method of order 4. A detailed description of its derivation can be found in [10].

$$
\begin{aligned}
c_{1} & =0, \quad c_{2}=c, \quad c_{3}=(3-\sqrt{3}) / 6, \quad c_{4}=(9+2 \sqrt{3}) / 23, \\
c_{5} & =(3+\sqrt{3}) / 6, \\
e_{1} & =(3-\sqrt{3}) / 4, \quad e_{2}=(3-\sqrt{3}) / 4-c, \quad e_{3}=1, \\
e_{4} & =(57+5 \sqrt{3}) / 92, \quad e_{5}=1 . \\
a_{21} & =c, \\
a_{32} & =(2-\sqrt{3}) /(12 c), \quad a_{31}=(3-\sqrt{3}) / 6-a_{32}, \\
a_{42} & =(2544-807 \sqrt{3}) /(13754 c), \quad a_{41}=(2781-647 \sqrt{3}) / 6877-a_{42}, \\
a_{43} & =(-90+1245 \sqrt{3}) / 6877, \\
a_{52} & =(-2+\sqrt{3}) /(12 c), \quad a_{51}=(-3+2 \sqrt{3}) / 9-a_{52}, \\
a_{53} & =1 / 5, \quad a_{54}=(57-5 \sqrt{3}) / 90, \\
b_{1} & =0, \quad b_{2}=0, \quad b_{3}=1 / 2, \quad b_{4}=0, \quad b_{5}=1 / 2 .
\end{aligned}
$$

The kernel condition (1.8) is satisfied, if the parameter $c$ satisfies $0<c \leqslant(3-\sqrt{3}) / 8$.

4. Some Additional Results. In the previous section we defined the $m$-stage Runge-Kutta method for equations of the form $y(x)=\int_{x_{0}}^{x} G(x, y(s)) d s$, and the extension to (1.1) is then based on (1.4).

Let now (1.1) be rewritten as

$$
y(x)=f\left(x_{0}\right)+\int_{x_{0}}^{x} \tilde{K}(x, s, y(s)) \cdot d s
$$

where

$$
\tilde{K}(x, s, y(s)):=\frac{f(x)-f\left(x_{0}\right)}{x-x_{0}}+K(x, s, y(s)) .
$$

The Runge-Kutta method for (4.1) is thus given by

$$
\begin{aligned}
& Y_{i}=f\left(x_{0}\right)+h \sum_{j=1}^{m} a_{i j} \tilde{K}\left(x_{0}+d_{i j} h, x_{0}+c_{j} h, Y_{j}\right) \quad(i=1, \ldots, m), \\
& y_{1}=f\left(x_{0}\right)+h \sum_{i=1}^{m} b_{i} \tilde{K}\left(x_{0}+e_{i} h, x_{0}+c_{i} h, Y_{i}\right),
\end{aligned}
$$

and this may be rewritten as (assuming that $d_{i j} \neq 0, e_{i} \neq 0$ )

$$
\begin{aligned}
Y_{i}= & f\left(x_{0}\right)+\sum_{j=1}^{m} \frac{a_{i j}}{d_{i j}} \cdot\left[f\left(x_{0}+d_{i j} h\right)-f\left(x_{0}\right)\right] \\
& +h \sum_{j=1}^{m} a_{i j} K\left(x_{0}+d_{i j} h, x_{0}+c_{j} h, Y_{j}\right) \quad(i=1, \ldots, m),
\end{aligned}
$$




$$
\begin{aligned}
y_{1}= & f\left(x_{0}\right)+\sum_{i=1}^{m} \frac{b_{i}}{e_{i}} \cdot\left[f\left(x_{0}+e_{i} h\right)-f\left(x_{0}\right)\right] \\
& +h \sum_{i=1}^{m} b_{i} K\left(x_{0}+e_{i} h, x_{0}+c_{i} h, Y_{i}\right) .
\end{aligned}
$$

If we choose $d_{i j}=e_{j}$ (which characterizes Bel'tyukov-type methods), we arrive at

$$
\left\{\begin{aligned}
k_{i}= & {\left[f\left(x_{0}+e_{i} h\right)-f\left(x_{0}\right)\right] } \\
& +h e_{i} K\left(x_{0}+e_{i} h, x_{0}+c_{i} h, f\left(x_{0}\right)+\sum_{j=1}^{m} \frac{a_{i j}}{e_{j}} \cdot k_{j}\right) \quad(i=1, \ldots, m), \\
y_{1}= & f\left(x_{0}\right)+\sum_{i=1}^{m} \frac{b_{i}}{e_{i}} \cdot k_{i} .
\end{aligned}\right.
$$

For the explicit case this form coincides with that given by Oulès [13]. As an example, consider the Bel'tyukov method (19) of [3] (compare also Example 3.7); if it is brought into the above form it reads as follows:

$$
\begin{aligned}
& k_{1}=f\left(x_{0}+h\right)-f\left(x_{0}\right)+h K\left(x_{0}+h, x_{0}, f\left(x_{0}\right)\right), \\
& k_{2}=f\left(x_{0}+h / 2\right)-f\left(x_{0}\right)+\frac{h}{2} \cdot K\left(x_{0}+h / 2, x_{0}+h / 2, f\left(x_{0}\right)+k_{1} / 2\right), \\
& k_{3}=f\left(x_{0}+h\right)-f\left(x_{0}\right)+h K\left(x_{0}+h, x_{0}+2 h / 3, f\left(x_{0}\right)+2 k_{1} / 9+4 k_{2} / 9\right), \\
& y_{1}=f\left(x_{0}\right)+\left(k_{1}+3 k_{3}\right) / 4 .
\end{aligned}
$$

This method was given by Oulès [12]; see also Aparo [1].

We now consider briefly the connection between collocation methods (in piecewise polynomial spaces) and Runge-Kutta methods of the form (1.3) for the Volterra equation (1.1). Suppose that (1.1) is solved by collocation, using piecewise polynomials $u$ of degree $m$ (which are permitted to have finite discontinuities at $x=x_{n}$, $n=1, \ldots, N)$; on $\left[x_{0}, x_{1}\right]$ the collocation points shall be $\left\{x_{0}+c_{i} h ; 0 \leqslant c_{1}<\cdots<\right.$ $\left.c_{m}<c_{m+1}=1\right\}$. The restriction of $u$ to $\left[x_{0}, x_{1}\right]$ is thus determined from

$$
\begin{array}{r}
u\left(x_{0}+c_{i} h\right)=f\left(x_{0}+c_{i} h\right)+h \int_{0}^{c_{i}} K\left(x_{0}+c_{i} h, x_{0}+s h, u\left(x_{0}+s h\right)\right) d s \\
(i=1, \ldots, m+1) .
\end{array}
$$

In general, the integrals in (4.5) have to be approximated by numerical quadrature. If we use (interpolatory) quadrature based on $\left\{c_{i} ; i=1, \ldots, m\right\}$, i.e., if (4.5) is replaced by

$$
\left\{\begin{array}{l}
Y_{i}=f\left(x_{0}+c_{i} h\right)+h \sum_{j=1}^{m} a_{i j} K\left(x_{0}+c_{i} h, x_{0}+c_{j} h, Y_{j}\right) \\
\text { with } \quad(i=1, \ldots, m+1), \\
y_{1}=u\left(x_{0}+h\right)=Y_{m+1} \quad\left(\text { note that } c_{m+1}=1\right),
\end{array}\right.
$$

then we obtain an $m$-stage implicit Pouzet method. (The case $m=1, c_{1}=1 / 2$, $c_{2}=1$, has been considered in Example $3.2\left(3.3^{\prime \prime}\right)$.) 
We note in passing that if the parameters $\left\{c_{i} ; i=1, \ldots, m\right\}$ are the zeros of $P_{m}(2 s-1)$ (Gauss points for $\left.(0,1)\right)$ then $(4.6)$ represents an $m$-stage implicit Pouzet method of order $2 \mathrm{~m}$.

Institut de Mathématiques

Université de Fribourg

Pérolles

CH-1700 Fribourg, Suisse

Institut für Angewandte Mathematik

Universität Heidelberg

Im Neuenheimer Feld 293

D-6900 Heidelberg 1, Germany

Institutt for Numerisk Mathematikk

Norges Tekniske Høgskole

N-7034 Trondheim-NTH, Norway

1. E. Aparo, "Sulla risoluzione numerica delle equazioni integrali di Volterra di seconda specie," Atti Accad. Naz. Lincei Rend. Cl. Sci. Fis. Mat. Natur., v. 26, 1959, pp. 183-188. (MR 23 \# B3159)

2. C. T. H. BAKER, The Numerical Treatment of Integral Equations, Clarendon Press, Oxford, 1977, pp. 849-864.

3. B. A. BeL'Tyuxov, "An analogue of the Runge-Kutta method for the solution of nonlinear integral equations of Volterra type," Differential Equations, v. 1, 1965, pp. 417-433. (MR 33 \#3479)

4. H. BRUNNER \& S. P. NøRSETT, Runge-Kutta Theory for Volterra Integral Equations of the Second Kind, Report 1/80, Dept. of Math., NTH-Trondheim, Norway, 1980.

5. J. C. BuTcher, "An algebraic theory of integration methods," Math. Comp., v. 26, 1972, pp. 79-106. (MR 46 \# 4378)

6. J. C. ButcheR, "Implicit Runge-Kutta and related methods," in Modern Numerical Methods for Ordinary Differential Equations (G. Hall and J. M. Watt, eds.), Clarendon Press, Oxford, 1976, pp. 136-151. (MR 57 \# 14454)

7. E. HAIRER \& G. WANNER, "Multistep-multistage-multiderivative methods for ordinary differential equations," Computing, v. 11, 1973, pp. 287-303. (MR 51 \# 14590)

8. E. Hairer \& G. WANNer, "On the Butcher group and general multi-value methods," Computing, v. 13, 1974, pp. 1-15. (MR 53 \#7037)

9. E. HAIRER, "Order conditions for numerical methods for partitioned ordinary differential equations," Numer. Math., v. 36, 1981, pp. 431-445.

10. E. HAIRER, "A fourth-order Bel'tyukov-type method for Volterra integral equations of the second kind." (In preparation.)

11. F. DE Hoog \& R. Weiss, "Implicit Runge-Kutta methods for second kind Volterra integral equations," Numer. Math., v. 23, 1975, pp. 199-213. (MR 51 \# 9549)

12. H. OulEs, "Sur la résolution numérique de l'équation intégrale de Volterra de seconde espèce," C. R. Acad. Sci. Paris, v. 250, 1960, pp. 964-965. (MR 22 1096)

13. H. OulEss, "Sur l'intégration numérique de l'équation integrale de Volterra de seconde espèce," C. R. Acad. Sci. Paris, v. 250, 1960, pp. 1433-1435. (MR 22 \#121)

14. P. Pouzet, "Etude en vue de leur traitement numérique des équations intégrales de type Volterra," Rev. Français Traitement Information (Chiffres), v. 6, 1963, pp. 79-112. (MR 27 \#2132) 